\title{
NON-DISCRIMINATING CRITERIA IN THE AHP: REMOVAL AND RANK REVERSAL
}

\author{
Diederik J.D. Wijnmalen \\ Operational Research and Business Management Division, Physics and Electronics Laboratory TNO \\ P.O. Box 96864, NL-2509 JG The Hague, The Netherlands \\ mailto:wijnmalen@fel.tno.nl \\ William C. Wedley \\ Faculty of Business Administration, Simon Fraser University \\ Burnaby, B. C. Canada, V5A 1S6 \\ mailto:wedley@sfu.ca
}

Keywords: AHP, non-discriminating criteria, rank reversal, weight adjustment

\begin{abstract}
Summary: A non-discriminating criterion is defined as a criterion where the decision-maker is indifferent among the alternatives. One would therefore expect the final rank order of the alternatives not to be affected by removing it. A previously published paper by J.S. Finan and W.J. Hurley (2002) showed, however, that in the Analytic Hierarchy Process removing such a criterion from a multilevel hierarchy can reverse rank. In this paper, we offer an explanation of this particular rank reversal phenomenon and show how it can be avoided. We do this by taking into account that there is a link between the normalisation and weighting processes, which suggests adjusting appropriate weights when removing criteria. Further, we discuss whether a non-discriminating criterion should be removed in the first place.
\end{abstract}

\section{Introduction}

In this paper, we address the phenomenon of non-discriminating criteria in an Analytic Hierarchy Process (AHP) hierarchy. A non-discriminating criterion is defined as a criterion where the decision-maker is indifferent among the alternatives when they are compared on that criterion. Since non-discriminating criteria do not differentiate between the alternatives, it is presumably safe to eliminate them from further consideration.

We take as a starting point a previously published paper by Finan \& Hurley (2002). In that paper, they denote a non-discriminating criterion as a "washcriterion" and investigate how the final rank order of the alternatives is affected by removing such a criterion from an AHP hierarchy. They differentiate between single-level hierarchies, that have only one level of criteria below the goal, and multilevel hierarchies, that have two or more criteria levels. They show that, assuming a perfectly consistent decision-maker, the final rank order of the alternatives is never affected by removing a non-discriminating criterion from a singlelevel hierarchy. But using a simple example of a two-level hierarchy, they show that in multilevel hierarchies, leaving out a non-discriminating criterion can reverse the final rank order. This is an interesting observation, since most literature on rank reversal in AHP relates to the addition or deletion of alternatives, not criteria (ref. Saaty (2000), and Belton \& Stewart (2002) for a brief summary).

Finan \& Hurley conclude that, since any hierarchy with multiple levels of criteria can be modelled as a hierarchy with a single level of criteria, the methods of synthesising a multilevel AHP hierarchy must be 
incorrect. Their comments add to the challenge of AHP methodology, but they provide no explanation for the defect nor any type of resolution.

The prime purpose of this paper is to show why rank reversal has occurred when synthesising the twolevel hierarchy and how it should have been avoided. We suggest that there is a necessary link between the normalisation and weighting processes and that Finan \& Hurley have failed to understand the meaning of a weight in a multiple level hierarchy in relation with the unit normalisation of the criteria weights. We show that proper adjustment of the appropriate weights avoids the rank reversal problem observed by Finan \& Hurley. We also show that it is not necessary to assume a perfectly consistent decision maker in order to prove that rank reversal will never occur when removing a non-discriminating criterion from a single-level hierarchy. We end by discussing whether or not one should remove non-discriminating criteria in the first place. In this paper, we restrict ourselves to additive synthesis using AHP's distributive mode (Saaty, 2000).

\section{Example of rank reversal in a two-level hierarchy}

Below follows the same example as Finan \& Hurley's of a hierarchy with two levels of criteria below the goal G: two main criteria $\left(\mathrm{C}_{1}, \mathrm{C}_{2}\right)$ on the first level and three sub-criteria of $\mathrm{C}_{1}\left(\mathrm{C}_{11}, \mathrm{C}_{12}, \mathrm{C}_{13}\right)$ and two subcriteria of $\mathrm{C}_{2}\left(\mathrm{C}_{21}, \mathrm{C}_{22}\right)$ on the second. Their respective local weights are shown in the boxes. The local priorities of two alternatives $A_{1}$ and $A_{2}$ are shown as well. Below the sub-criterion boxes, the global weights of the sub-criteria are shown in italics.

Figure 1: Example of a hierarchy with one non-discriminating sub-criterion

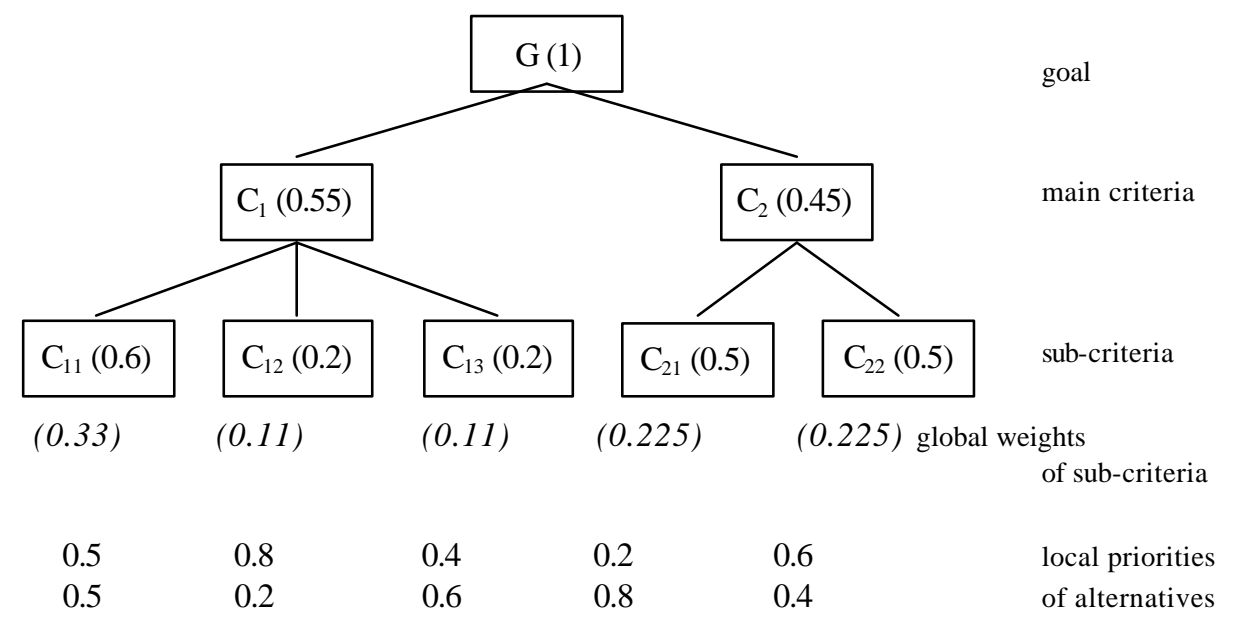

First, we compute the initial composite priorities of the alternatives as follows, using additive synthesis:

$$
\begin{aligned}
& \mathrm{A}_{1}=\{0.5 * 0.6+0.8 * 0.2+0.4 * 0.2\} * 0.55+\{0.2 * 0.5+0.6 * 0.5\} * 0.45=0.477 \\
& \mathrm{~A}_{2}=\{0.5 * 0.6+0.2 * 0.2+0.6 * 0.2\} * 0.55+\{0.8 * 0.5+0.4 * 0.5\} * 0.45=0.523
\end{aligned}
$$

From this follows that $A_{2}$ is $0.523 / 0.477=1.096$ times preferred to $A_{1}$.

We notice that $\mathrm{C}_{11}$ does not discriminate between the alternatives; they are equally attractive on that subcriterion. If $\mathrm{C}_{11}$ is removed from the hierarchy and $\mathrm{C}_{12}$ and $\mathrm{C}_{13}$ are re-normalised to the unit sum, $\mathrm{C}_{12}$ and $\mathrm{C}_{13}$ 
will get higher weights: 0.5 each. Without any other adjustment, the new composite priorities of the alternatives with $\mathrm{C}_{11}$ removed are as follows (these are Finan \& Hurley's findings):

$$
\begin{aligned}
& \mathrm{A}_{1}=\left\{0.8^{*} 0.5+0.4 * 0.5\right\}^{*} 0.55+\{0.2 * 0.5+0.6 * 0.5\}^{*} 0.45=0.51 \\
& \mathrm{~A}_{2}=\left\{0.2 * 0.5+0.6^{*} 0.5\right\}^{*} 0.55+\left\{0.8 * 0.5+0.4^{*} 0.5\right\}^{*} 0.45=0.49
\end{aligned}
$$

From this follows that now $\mathrm{A}_{1}$ is $0.51 / 0.49=1.041$ times preferred to $\mathrm{A}_{2}$; their ranks are reversed compared with the initial result above.

\section{No rank reversal in a single-level hierarchy}

In AHP synthesis, $\mathbf{i}$ is possible to first compute the global weights of the sub-criteria by successive multiplication of the local criteria weights and then multiply the local alternative priorities with those global weights. This boils down to creating a single-level hierarchy where only the lowest level of criteria (the former sub-criteria in our example) are shown with their global weights. In such a revised hierarchy, there would be no difference between local and global weights. The global weights of our example are shown in italics in Figure 1.

Taking $\mathrm{C}_{11}$ into account, the composite priorities are as follows:

$$
\begin{aligned}
& \mathrm{A}_{1}=0.5 * 0.33+0.8^{*} 0.11+0.4^{*} 0.11+0.2 * 0.225+0.6^{*} 0.225=0.477 \\
& \mathrm{~A}_{2}=0.5 * 0.33+0.2 * 0.11+0.6^{*} 0.11+0.8 * 0.225+0.4^{*} 0.225=0.523
\end{aligned}
$$

The results are of course identical to the initial results in section $2 ; \mathrm{A}_{2}$ is $0.523 / 0.477=1.096$ times preferred to $\mathrm{A}_{1}$.

Next, we remove $\mathrm{C}_{11}$, the non-discriminating sub-criterion, and compute new composite priorities by simply subtracting $0.5 * 0.33=0.165$ from the previous ones. Thus:

$$
\begin{aligned}
& \mathrm{A}_{1}=0.477-0.165=0.312 \\
& \mathrm{~A}_{2}=0.523-0.165=0.358
\end{aligned}
$$

The new ratio is $0.358 / 0.312=1.147$, thereby heightening the difference between the two alternatives and making $\mathrm{A}_{2}$ more pronounced as the best alternative. We observe that there is no rank reversal with this process.

We could have first re-normalised the remaining global weights and then computed the weighted sums, as follows:

$$
\begin{aligned}
& \mathrm{A}_{1}=0.8 * 0.1642+0.4 * 0.1642+0.2 * 0.3358+0.6 * 0.3358=0.4657 \\
& \mathrm{~A}_{2}=0.2 * 0.1642+0.6 * 0.1642+0.8 * 0.3358+0.4 * 0.3358=0.5343
\end{aligned}
$$

The composite priorities are of course higher owing to the re-normalisation (which heightens the weights), but their rank order is preserved and A2 is still 1.147 more preferred than A1.

We shall now show that in a single-level hierarchy rank reversal will never occur when removing a nondiscriminating criterion, even if the decision-maker is not perfectly consistent ${ }^{1}$.

Suppose that we have a set $J=\{0,1, \ldots, n\}$ of $n+1$ criteria in an AHP hierarchy with one level of criteria below the goal. The non-discriminating criterion is indexed by 0 . The reduced set is denoted by $\underline{J}=\{1, \ldots$,

\footnotetext{
${ }^{1} \mathrm{We}$ are indebted to an anonymous researcher for pointing out to us that consistency is not required.
} 
$n\}$. We have criteria weights $c_{j}(j=0, \ldots, n)$ for $J$, with $\sum_{j} c_{j}=1$, and $\underline{c}_{j}(j=1, \ldots, n)$, with $\sum_{j} \underline{c}_{j}=1$ for the reduced set $\underline{J}$. Assuming that we already know the values of $c_{j}$, with all $c_{j}<1$, then it is reasonable to conclude that $c_{i} / c_{j}=\underline{c}_{i} / \underline{c}_{j}$ for $i, j \in\{1, \ldots, n\}$. Define a constant $\theta$ such that $\underline{c}_{i} / c_{i}=\underline{c}_{j} / c_{j}=\theta$ for $i, j \in\{1, \ldots$, $n\}$.

From $\sum_{j=1}^{n} \underline{c}_{j}=1=\theta \sum_{j=1}^{n} c_{j}=\theta\left(1-c_{0}\right)$, it follows that $\theta=1 /\left(1-c_{0}\right)$, and therefore that $\underline{c}_{j}=c_{j} /\left(1-c_{0}\right)$ for $j \in$ $\{1, \ldots, n\}$.

Let the local priority of an alternative $x(x=1, \ldots, m)$ on a criterion $i$ be denoted by $u_{x i}$ and its composite priority for the set $J$ be denoted by $w_{x}$ and for the reduced set $\underline{J}$ by $\underline{w}_{x}$. In particular, we have $u_{x 0}=1 / m$ for all $\mathrm{x} \in\{1, \ldots, m\}$ since the criterion indexed by 0 is non-discriminatory. Using additive synthesis, we compute the following difference between the composite priorities of two alternatives $x$ and $y$ for the full set $J$ (see also Finan \& Hurley, 2002):

$$
\begin{aligned}
w_{x}-w_{y} & =\sum_{k=0}^{n} c_{k} u_{x k}-\sum_{k=0}^{n} c_{k} u_{y k} \\
& =\left[c_{0} / m+\sum_{k=1}^{n}\left(1-c_{0}\right) \underline{c}_{k} u_{x k}\right]-\left[c_{0} / m+\sum_{k=1}^{n}\left(1-c_{0}\right) \underline{c}_{k} u_{y k}\right] \\
& =\left(1-c_{0}\right)\left\{\sum_{k=1}^{n} \underline{c}_{k} u_{x k}-\sum_{k=1}^{n} \underline{c}_{k} u_{y k}\right\} \\
& =\left(1-c_{0}\right)\left\{\underline{w}_{x}-\underline{w}_{y}\right\}
\end{aligned}
$$

Since $\left(1-\mathrm{c}_{0}\right)>0$, the signs of $\left(w_{x}-w_{y}\right)$ and $\left(\underline{w}_{x}-\underline{w}_{y}\right)$ are the same, and, therefore, the rank order is not affected by removing the non-discriminating criterion. Note that we have never used the assumption of a perfectly consistent decision-maker. The above can of course be extended to removal of more than one non-discriminating criterion.

\section{Adjustment to avoid rank reversal in a multilevel hierarchy}

As noted in section 2, rank reversal can occur in a multilevel hierarchy if the non-discriminating subcriterion is removed and local criteria weights are re-normalised. Had we not re-normalised criteria weights after removing the non-discriminating sub-criterion, then the composite results would have been:

$$
\begin{aligned}
& \mathrm{A}_{1}=\{0.8 * 0.2+0.4 * 0.2\} * 0.55+\{0.2 * 0.5+0.6 * 0.5\}^{*} 0.45=0.312 \\
& \mathrm{~A}_{2}=\{0.2 * 0.2+0.6 * 0.2\} * 0.55+\{0.8 * 0.5+0.4 * 0.5\} * 0.45=0.358
\end{aligned}
$$

We notice that these results and their ratios are identical to the ones in (4) obtained by simply subtracting each alternative's global priority on the non-discriminating sub-criterion from their composite priorities. In section 2, the local unit normalisation after removing $\mathrm{C}_{11}$ obviously distorts the ratio of the global weights of $\mathrm{C}_{1}$ 's criteria set with respect to those of the $\mathrm{C}_{2}$ set, which keep their original weights. The result of the distortion is the rank reversal shown in section 2. So, why did it go wrong with the synthesis using renormalised local weights?

What one should take into consideration is the meaning of a criterion's weight. As shown by Schoner et. al. (1997) and Choo et. al. (1999), there is a necessary link between the normalisation process and the weighting process. Finan \& Hurley have used unit sum normalisation of local weights without realising that the unit of that sum changes with the removal of the sub-criterion. Initially in that procedure, all local weights and priorities are normalised with respect to the totality of each set they are part of; the totality thus gets the unit value for many local ratio scales. In this respect, the local weights that sum to unity below each node can be visualised as a whole series of little hierarchies (node and sub-nodes) that have not yet been synthesised into the overall hierarchy (and an overall ratio scale). The unit weight of each 
sub-criterion pertains to the totality of the alternatives it is covering; the unit weight of each main criterion pertains to the totality of the sub-criteria it is covering.

It is subsequently the process of synthesis via hierarchical weighting which transforms the local weights into global weights that are in terms of a unit of the whole hierarchy. In effect, the former unit value that each node represented for the totality of directly covered items is re-scaled to be in terms of an overall hierarchical unit representing all alternatives and all criteria. Although this proportional transformation of local weights yields a new unit of measure, the global weights are still weighing the relative imp ortance of the totalities they are referring to. The global weight of each sub-criterion pertains to the totality of the alternatives it is covering; similarly, the global weight of each main criterion pertains to the totality of the sub-criteria and altematives it is covering.

When deriving local criteria weights, it is important to keep in mind the totality of the items below as a reference. The weights should be adjusted accordingly when the totality changes due to a change in either the set of alternatives or the set of sub-criteria, while getting the unit value again. Examples of changing the set of alternatives can be found in the existing literature on AHP rank reversal (Saaty (2000), Belton \& Stewart (2002)). A change in the set of sub-criteria is the case in Finan \& Hurley's example, reproduced in our section 2. Leaving out $C_{11}$ changes $C_{1}$ 's set of sub-criteria; the new set is now $0.2+0.2=0.4$ of its former self. The main criterion $\mathrm{C}_{1}$ now represents a different totality of sub-criteria below. Accordingly, the weight of $\mathrm{C}_{1}$ should be adjusted to $0.55 * 0.4=0.22$ in order to safely re-normalise the remaining local weights of its sub-set to the unit sum of the total hierarchy.

The adjusted criterion weight for $C_{1}(0.22)$ remains commensurate to $C_{2}(0.45)$, although they do not sum to one. Synthesis using the new, normalised local weights of $\mathrm{C}_{12}$ and $\mathrm{C}_{13}$ (both 0.5 ) and the adjusted weight for $\mathrm{C}_{1}$ produces:

$$
\begin{aligned}
& \mathrm{A}_{1}=\left\{0.8^{*} 0.5+0.4^{*} 0.5\right\} * 0.22+\left\{0.2 * 0.5+0.6^{*} 0.5\right\} * 0.45=0.312 \\
& \mathrm{~A}_{2}=\left\{0.2 * 0.5+0.6^{*} 0.5\right\} * 0.22+\left\{0.8^{*} 0.5+0.4 * 0.5\right\} * 0.45=0.358
\end{aligned}
$$

The new values of the global weights can be computed using $\mathrm{C}_{1}$ 's adjusted weight and the new, normalised local weights of $\mathrm{C}_{12}$ and $\mathrm{C}_{13}$ (both 0.5 ):

$$
\begin{aligned}
& C_{12}=C_{13}=0.22 * 0.5=0.11 \\
& C_{21}=C_{22}=0.45 * 0.5=0.225
\end{aligned}
$$

The global weights of $\mathrm{C}_{12}, \mathrm{C}_{13}, \mathrm{C}_{21}$ and $\mathrm{C}_{22}$ have not changed and neither have the local priorities of the alternatives. Synthesis again yields composite priorities of 0.312 for $A_{1}$ and 0.358 for $A_{2}$ with $A_{2} 1.147$ times more preferred than $\mathrm{A}_{1}$, therefore not showing rank reversal.

Had $C_{1}=0.22$ and $C_{2}=0.45$ been re-normalised to sum to one, then the result $C_{1}=0.328$ and $C_{2}=0.672$ would still be commensurate, but with a different unit of measure. Synthesis using the new, normalised local weights of $\mathrm{C}_{12}$ and $\mathrm{C}_{13}$ (both 0.5 ) and the re-normalised weight for $\mathrm{C}_{1}$ and $\mathrm{C}_{2}$ (after adjustment of $\mathrm{C}_{1}$ 's weight) produces:

$$
\begin{aligned}
& \mathrm{A}_{1}=\left\{0.8^{*} 0.5+0.4^{*} 0.5\right\}^{*} 0.328+\left\{0.2 * 0.5+0.6^{*} 0.5\right\}^{*} 0.672=0.4657 \\
& \mathrm{~A}_{2}=\left\{0.2^{*} 0.5+0.6^{*} 0.5\right\}^{*} 0.328+\left\{0.8 * 0.5+0.4^{*} 0.5\right\}^{*} 0.672=0.5343
\end{aligned}
$$

With global weights, computed from adjusted, fully re-normalised local weights, the results would be identical to those in (8). 
Table 1: Summary of results using different procedures (RR: rank reversal)

\begin{tabular}{|c|c|c|c|}
\hline synthesis procedure & $\begin{array}{c}\text { results } \\
\text { reference }\end{array}$ & $\begin{array}{l}\text { composite } \\
\text { priorities }\end{array}$ & $\begin{array}{l}\text { rank order, } \\
\text { ratio }\end{array}$ \\
\hline $\begin{array}{l}\text { full synthesis including } \mathrm{C}_{11} \text {, } \\
\text { using either local or global weights }\end{array}$ & $(1),(3)$ & $\begin{array}{l}\mathrm{A}_{1}=0.477 \\
\mathrm{~A}_{2}=0.523\end{array}$ & $\mathrm{~A}_{2}>\mathrm{A}_{1}(1.096)$ \\
\hline $\begin{array}{l}\text { synthesis excluding } \mathrm{C}_{11} \text {, } \\
\text { using re-normalised (with respect to } \mathrm{C}_{1} \text { ) local weights } \\
\text { (usual procedure of AHP's additive synthesis) }\end{array}$ & (2) & $\begin{array}{l}\mathrm{A}_{1}=0.51 \\
\mathrm{~A}_{2}=0.49\end{array}$ & $\begin{array}{l}\mathrm{A}_{1}>\mathrm{A}_{2}(1.041) \\
\mathbf{R R}\end{array}$ \\
\hline $\begin{array}{l}\text { synthesis excluding } \mathrm{C}_{11} \text {, } \\
\text { using original composite priorities and } \\
\text { not re-normalised global weights }\end{array}$ & (4) & $\begin{array}{l}\mathrm{A}_{1}=0.312 \\
\mathrm{~A}_{2}=0.358\end{array}$ & $\mathrm{~A}_{2}>\mathrm{A}_{1}(1.147)$ \\
\hline $\begin{array}{l}\text { synthesis excluding } \mathrm{C}_{11} \text {, } \\
\text { using re-normalised global weights }\end{array}$ & (5) & $\begin{array}{l}\mathrm{A}_{1}=0.4657 \\
\mathrm{~A}_{2}=0.5343\end{array}$ & $\mathrm{~A}_{2}>\mathrm{A}_{1}(1.147)$ \\
\hline $\begin{array}{l}\text { synthesis excluding } \mathrm{C}_{11} \text {, } \\
\text { using not re-normalised local weights }\end{array}$ & (6) & $\begin{array}{l}\mathrm{A}_{1}=0.312 \\
\mathrm{~A}_{2}=0.358\end{array}$ & $\mathrm{~A}_{2}>\mathrm{A}_{1}(1.147)$ \\
\hline $\begin{array}{l}\text { synthesis excluding } C_{11} \text {, } \\
\text { using adjusted re-normalised (with respect to } C_{1} \text {, but } \\
\text { not } G \text { ) local weights } \\
\text { (identical to using adjusted not re-normalised global } \\
\text { weights) }\end{array}$ & (7) & $\begin{array}{l}\mathrm{A}_{1}=0.312 \\
\mathrm{~A}_{2}=0.358\end{array}$ & $\mathrm{~A}_{2}>\mathrm{A}_{1}(1.147)$ \\
\hline $\begin{array}{l}\text { synthesis excluding } C_{11} \text {, } \\
\text { using adjusted re-normalised (with respect to both } C_{1} \\
\text { and } G \text { ) local weights } \\
\text { (identical to using adjusted re-normalised global } \\
\text { weights) }\end{array}$ & (8) & $\begin{array}{l}\mathrm{A}_{1}=0.4657 \\
\mathrm{~A}_{2}=0.5343\end{array}$ & $\mathrm{~A}_{2}>\mathrm{A}_{1}(1.147)$ \\
\hline
\end{tabular}

\section{Removal of non-discriminating criteria}

Since the removal of non-discriminating criteria does not alter the best choice solution, should they be removed in the first place? The answer depends upon the purpose of the problem and whether the accentuation of differences is worthwhile.

If the problem involves resource allocation such as dividing a bonus pool between several recipients, the problem is not of the "best choice" type. In such closed resource allocation situations, removing a nondiscriminating criterion would give different composite ratios and therefore a different distribution of bonuses, probably with dissension and undesirable consequences. In effect, the elimination of a criterion would be a re-definition of how the resources are distributed. Those receiving lower bonuses could legitimately argue that if the non-discriminating criterion was relevant to start with, then it is relevant to the end and should not be removed.

On the other hand, if the situation is a "best choice" problem for which we only desire an ordinal solution, the elimination of non-discriminating criteria can give greater emphasis to the best choice. If done indiscriminately, such re-framing could make the best choice appear vastly superior on an inconsequential 
set of remaining criteria. For the purpose of scientific integrity, our preference is to diligently define and structure the problem at the outset and then use all relevant criteria to make the decision, including those that turn out to be non-discriminatory.

\section{Final remarks and conclusion}

Rarely are weights adjusted upon a change of a set of elements in an AHP hierarchy. This is perhaps not surprising as AHP's axiom 3, the " independence axiom" (Saaty (2000)), does not formally require criteria weights to be derived in relation to lower-level hierarchy elements and their normalisation. The independence axiom states that elements on a specific hierarchy level are dependent on their parentelements on the next higher hierarchy level, but independent of their child-elements (criteria or alternatives) on the next lower level.

We believe, however, that it is better to assume dependence and deal with it properly than to assume independence and face phenomena like rank reversal that are difficult to justify or explain. In this paper, we have shown that assuming dependence and acting accordingly prevents undue rank reversal when a nondiscriminating criterion is removed from the hierarchy. This situation is particularly striking and has the flavour of the rank reversal problem of Belton \& Gear (1983) where a copy of an existing alternative was added to the set of alternatives. In both cases, the totality of a set of hierarchy elements was changed, thereby changing the unit of that totality when re-normalising to the unit sum. Incidentally, that totality will change regardless of the non-discriminatory nature of a criterion being removed or the identical nature of an alternative being added. As soon as one or more of the sets of hierarchy elements are changed by adding or removing (any) elements, the appropriate local weights must be re-considered and probably adjusted to maintain commensurateness and thus prevent ranks from reversing. In case of criteria that do not discriminate between alternatives, an alternative approach is to first compute global weights and then remove non-discriminating criteria and their global weights from synthesis.

It should be pointed out that, so far, we have used the weaker requirement of rank preservation rather than ratio preservation that could be applied to AHP. As shown above, the deletion of a criterion can cause composite ratios to change, although proper weight adjustment preserves rank. However, if an alternative is added or deleted, the structural adjustment we have suggested would not have resulted in a change in ratios. The reason for this differential effect is that the global priorities of the alternatives on each criterion are in commensurate units. If the alternatives are discrete and independent of one another, then summation across all criteria does not upset the ratio between existing alternatives. If, however, we sum across different sub-sets of criteria, the results will be different ratios. In effect, each addition or deletion of criteria presents a new evaluation problem whereas addition or deletion of an alternative just changes the choice set in the same evaluation problem. In both cases, however, the appropriate weights must be reconsidered and most likely adjusted in order to maintain the integrity of the ranks.

\section{References}

Belton, V. and Gear, T. (1983), “ On a shortcoming of Saaty's method of analytic hierarchies”, Omega, 11, 228-230.

Belton, V. and Stewart, T.J. (2002), Multiple Criteria Decision Analysis, An Integrated Approach, Boston: Kluwer.

Choo, E.U., Schoner, B. and Wedley, W.C. (1999), “ Interpretation of criteria weights in multi-criteria decision making”, Computers and Industrial Engineering Journal, 37; 527-541.

Finan, J.S. and Hurley, W.J. (2002), “The analytic hierarchy process: can wash criteria be ignored?” Computers \& Operations Research, 29; 1025-1030. 
Saaty, T.L. (2000), Fundamentals of Decision-Making and Priority Theory with the Analytic Hierarchy Process, Pittsburgh: RWS Publications.

Schoner, B., Choo, E.U. and Wedley, W.C. (1997), “A comment on 'Rank disagreement: A comparison of multi-criteria methodologies"”, Journal of Multi-Criteria Decision Analysis, 6; 197-200. 